\title{
Impaired temporal discrimination within the attentional blink
}

\author{
Bettina Rolke, Karin M. Bausenhart, And Rolf Ulrich \\ University of Tübingen, Tübingen, Germany
}

\begin{abstract}
Yeshurun and Levy (2003) reported that temporal discrimination performance of a visual stimulus benefits when spatial attention is oriented away from its location. In the present study, we investigated whether this negative influence of attention on temporal discrimination performance is restricted to transient spatial attention or might generalize to other paradigms of attention. We employed the attentional blink $(\mathrm{AB})$ paradigm and required either a spatial (Experiment 1) or a temporal discrimination task (Experiment 2). The results of both experiments revealed a performance decrement if attention was temporally unavailable during the $\mathrm{AB}$ and a recovery with increasing attentional availability. Thus, contrary to the results of Yeshurun and Levy, the absence of attention decreased temporal discrimination performance in this paradigm. We hypothesize that attention which operates at different processing levels might exert differential effects on temporal stimulus processing.
\end{abstract}

Although a variety of different environmental stimuli are continuously absorbed by our senses, selective attention allows a subset of these stimuli to enter a deeper processing and an access to memory. Since about 40 years, the phenomenon of selective attention has been intensively investigated within cognitive psychology (see Neisser, 1967), and the functional role of selective attention is often compared with a filter mechanism. In this sense, attention enhances the processing of selected stimuli and attenuates (Treisman, 1960), or hinders (see, e.g., Broadbent, 1958) the processing of unselected stimuli.

This view of attention is supported by a bulk of spatial cuing studies showing that the allocation of visual attention to a target location facilitates the processing of a stimulus that occurs at this location (cf. Pashler, 1998). For example, attending a specific location enhances perceptual accuracy at this location in a variety of tasks, such as the discrimination of line length (Bonnel, Possamaï, \& Schmitt, 1987), line orientation (see, e.g., Cheal, Lyon, \& Hubbard, 1991; Downing, 1988), orientation of Gabor patches (Yeshurun \& Carrasco, 2000), Landolt squares (Yeshurun \& Carrasco, 1999), and letters (see, e.g., Henderson, 1991, 1996). These studies are in agreement with the notion that visual attention facilitates stimulus processing.

There are, however, two exceptions to this rule which show that the role of attention on stimulus processing is much more complex. First, Yeshurun and Carrasco (1998) found that visual attention improves the detection of texture segregation at the periphery, but hampers detection at more central locations. The authors explained this finding by assuming that attention improves the spatial resolution of the visual system and therefore hinders processing of specific stimulus features which require less resolution.
Since the spatial resolution of the visual system naturally differs between central and peripheral locations, attention differentially affects performance in response to stimuli at these locations. Second, an even more dramatic impairment of attentive stimulus processing was reported by Yeshurun and Levy (2003). The authors provided surprising evidence that selective attention exerts differential effects on the processing of spatial and temporal stimulus properties. In a series of experiments, these authors adapted a spatial cuing paradigm (Posner, Snyder, \& Davidson, 1980) to investigate the influence of spatial attention on the processing of spatial and temporal stimulus properties.

In one experiment, Yeshurun and Levy (2003) asked their participants to perform a spatial task (to detect a gap in a Landolt square). To manipulate attentional processing, a spatial cue appeared shortly before the presentation of the target stimulus. In the valid condition, this cue shifted visual attention to the location at which the stimulus was presented. In the neutral condition, however, the cue did not restrict attention to a particular location. Consistent with other studies on visual attention, the detection of the spatial gap was improved in the valid condition replicating the common effect of visual attention on spatial stimulus properties. In another experiment, Yeshurun and Levy (2003) employed the same cuing paradigm but now investigated the influence of attention on the processing of temporal stimulus properties. This time, their participants performed a temporal discrimination task. In some trials, two successive light flashes separated by a brief interval were presented to the same location in the display. In the remaining trials, however, a single continuous flash was presented. Participants were asked to discriminate be-

B. Rolke, bettina.rolke@uni-tuebingen.de 
tween both types of trials. Surprisingly, the detection of the temporal gap was impaired in the valid condition. This result indicates a negative influence of spatial attention on temporal stimulus processing.

Recently, Rolke, Dinkelbach, Hein, and Ulrich (in press) replicated this effect with an alternative arrangement of cues. These authors replaced the neutral cue employed in the study of Yeshurun and Levy (2003) by an invalid cue, which was physically identical to the valid one. In line with the results of Yeshurun and Levy, temporal gap detection was impaired when attention was oriented toward the stimulus location. In addition, Hein, Rolke, and Ulrich (2006) have successfully generalized this negative effect of attention to another temporal task in which participants were asked to discriminate the temporal order of two spatially adjacent dots. In a series of three experiments and in accordance with Yeshurun and Levy's result, they have shown that automatically oriented visual attention impairs discrimination performance. Therefore, this negative effect of visual spatial attention on temporal discrimination turns out to be a robust phenomenon.

Since this effect of attention on temporal stimulus processing provides new insights into the functional role of attention, it is of theoretical importance to investigate whether it is restricted to spatial attention or whether it generalizes to other task situations. More specifically, all the studies reporting the negative effect of attention on temporal stimulus processing have manipulated transient attention. It has been assumed, however, that transient attention influences rather early perceptual processing stages (Briand \& Klein, 1987; Luck, et al., 1994; Nakayama \& Mackeben, 1989; Riggio \& Kirsner, 1997). In fact, these studies are consistent with the view that the effect of visual attention is entirely restricted to early levels of visual processing, and it is not clear whether attentional influences at a later processing level may also affect the processing of temporal stimulus properties.

In the present study, we investigated whether an attentional manipulation at a later processing stage influences temporal discrimination performance. To manipulate the allocation of attention at a later processing level, we used the attentional blink paradigm and employed a rapid serial visual presentation (RSVP) method. The task in this paradigm is to identify two target stimuli which are embedded in a stream of rapidly presented (presentation rate: $10-20 \mathrm{~Hz}$ ) distractor stimuli. If participants correctly identify the first target (T1) in this RSVP stream of stimuli, the processing of a second target (T2) is impaired (e.g., Broadbent \& Broadbent, 1987; Raymond, Shapiro, \& Arnell, 1992). This deficit in processing T2 lasts several hundred msec after T1 presentation and has been labeled the attentional blink (AB).

Most important for the purpose of the present study, the $\mathrm{AB}$ presumably arises at a postperceptual processing level. This late locus of the $A B$ is supported by several lines of evidence. First, in the standard AB paradigm, one usually compares performance in a dual-task situation, when T1 and T2 have to be reported, with single-task performance, when only $\mathrm{T} 2$ has to be reported and the participants are instructed to ignore $\mathrm{T} 1$. Whereas both conditions are equivalent in terms of the visual input, they differ in terms of attentional and processing requirements. The typical result reveals a deficit in reporting T2 which is usually restricted to the dual-task condition (e.g., Raymond, et al., 1992; Ross \& Jolicœur, 1999). If perceptual factors would contribute to the $\mathrm{AB}$, the processing deficit should also occur in the single-task condition. Therefore, this comparison between dual-task and single-task ascertains that the processing deficit for $\mathrm{T} 2$ is not due to sensory factors such as low-level masking, but instead reflects limited capacity processes associated with attending to T1 in the RSVP.

Second, the late locus of the $\mathrm{AB}$ furthermore is supported by the result that an impaired detection performance during the $\mathrm{AB}$ is not accompanied by a suppression of early evoked potentials (Vogel, Luck, \& Shapiro, 1998). This result contrasts with the results emerging from exogenous spatial attention studies. In these studies, early evoked potentials are reduced by the absence of attention (Luck \& Girelli, 1998; Luck et al., 1994; Mangun \& Hillyard, 1991). Thus, the result that early evoked potentials are uninfluenced during the $\mathrm{AB}$ suggests that the perceptual processing of blinked stimuli remains unaffected. Moreover, the demonstration of a semantic processing within the $\mathrm{AB}$ (Luck, Vogel, \& Shapiro, 1996; Maki, Frigen, \& Paulson, 1997; Rolke, Heil, Streb, \& Hennighausen, 2001; Shapiro, Driver, Ward, \& Sorensen, 1997) strongly argues for a postsemantic processing deficit. Taken all the evidence about the locus of the $\mathrm{AB}$ together, it can be assumed that the stimuli which are presented during the $\mathrm{AB}$ time interval are perceptually processed, but do not reach a stable level of representation that would allow memory retrieval and conscious access.

These properties of the $\mathrm{AB}$ are combined to the most prominent model of the $\mathrm{AB}$, which constitutes a bottleneck model (but see Isaak, Shapiro, \& Martin, 1999; Raymond, Shapiro, \& Arnell, 1995; Shapiro, Raymond, $\&$ Arnell, 1994 for different accounts). This bottleneck model (Arnell \& Jolicœur, 1999; Jolicœur, Dell'Acqua, \& Crebolder, 2001; Ruthruff \& Pashler, 2001; see also Chun \& Potter, 1995) assumes that all RSVP items pass though a preattentional processing stage, but for later report they have to be consolidated in a more stable short-term memory representation by means of a bottleneck process. This bottleneck process operates serially and if it is occupied by the processing required for one task, then it cannot process another task. According to standard explanations of dualtask interference (see, e.g., Pashler \& Johnston, 1989), the $\mathrm{AB}$ arises from this bottleneck processing stage, that is, at a postperceptual stage. Since processing duration of T1 exceeds its physical presence at high presentation rates in RSVP, the bottleneck processing stage is still occupied with $\mathrm{T} 1$ processing when $\mathrm{T} 2$ is presented at short stimulus onset asynchronies (SOA). As a consequence, the representation of T2 has to remain in the preattentional processing stage until T1 bottleneck processing has finished. While the instable $\mathrm{T} 2$ representation waits for consolidation, however, it is likely to decay or may be overwritten by the following items at short SOA. This bottleneck model of the AB is supported by behavioral (Chun \& Potter, 1995; Crebolder, Jolicœur, \& McIlwaine, 2002) as well as elec- 
trophysiological evidence (Vogel \& Luck, 2002). On the whole, the empirical evidence concerning the locus of the $\mathrm{AB}$ effect strongly suggests that the $\mathrm{AB}$ effect arises at a postperceptual processing level. Therefore, the $\mathrm{AB}$ modulation provides a useful tool to investigate late-attentional effects on temporal stimulus processing.

The aim of the present study is to examine whether there is an $\mathrm{AB}$ for temporal stimulus properties or not. Although there are several studies which have shown that an $\mathrm{AB}$ occurs for the processing of a variety of stimulus properties, surprising few studies have investigated temporal stimulus processing within the RSVP. For example, an AB has been reported for letters (Chun \& Potter, 1995; Raymond et al., 1992), colors (Ross \& Jolicœur, 1999), and words (Luck et al., 1996; Rolke et al., 2001; Shapiro et al., 1997). In addition, the AB is also present for auditory stimuli and for cross-modal stimulus arrangements indicating it is not restricted to a specific stimulus modality (Arnell \& Jolicœur, 1999; Arnell \& Larson, 2002; Dell'Acqua, Turatto, \& Jolicœur, 2001). Since all these mentioned stimulus properties are nontemporal, in line with spatial attention studies, one should expect an impaired processing performance if attentional resources are decreased during the $\mathrm{AB}$.

There are only a few studies, however, which have investigated the processing of temporal stimulus properties within the RSVP. In a series of experiments, Shapiro et al. (1994) examined the relationship of attentional demands of various target tasks on the AB. In some experiments of the study, T1 task required the participants to identify or to detect an object, such as a letter within a stream of distractor letters. In other experiments, however, participants were instructed to detect a temporal gap within the RSVP stream or to judge the duration of this gap as short or long. $\mathrm{T} 2$ task in all experiments was to detect the presence of an "X" within the RSVP stream. Whereas the authors obtained a pronounced $\mathrm{AB}$ for object tasks, the $\mathrm{AB}$ was not elicited when the target was a temporal gap. Specifically, the absence of an $\mathrm{AB}$ was based on the result that there was no influence of SOA on T2 detection performance. Furthermore, there was also no effect of condition - that is, T2 detection performance did not differ between a dual-task, in which participants attended to T1 and T2 and a single-task, in which participants only had to detect $\mathrm{T} 2$. To exclude the possibility that temporal tasks generally required less attention and therefore did not elicit an $\mathrm{AB}$, the authors calculated $d^{\prime}$ as a measure of T1 task processing difficulty. This analysis revealed that the temporal tasks were considerably more difficult than the object tasks. Therefore, the absence of an $\mathrm{AB}$ in the temporal tasks can not be explained by task demands. Since no AB is elicited when the target is defined as temporal event, the authors concluded that only the processing of physically patterned objects as targets will cause an $\mathrm{AB}$.

To further specify the role of the temporal T1 task processing on the AB, Sheppard, Duncan, Shapiro, and Hillstrom (2002) conducted another series of experiments. In their first experiment, they replicated the previous results, namely, the absence of the $\mathrm{AB}$ when $\mathrm{T} 1$ task required a temporal discrimination. In a second experiment, the $\mathrm{AB}$ was again absent when the T1 task was to discriminate the duration of a $\mathrm{T} 1$ letter presented for a longer duration than the other letters in the stream. The AB was again observed in their third experiment, however, when the task was to identify a T1 letter which lasted longer than the other RSVP stimuli. Thus, the authors assumed that not the presence of a patterned object as T1 itself is crucial for the occurrence of the AB. Instead, they argued that something about the $\mathrm{T} 1$ duration judgment task leads to the elimination of the $\mathrm{AB}$. In their fourth experiment, the authors reported the reinstatement of the $\mathrm{AB}$ when participants discriminated very long temporal gaps (440 vs. $880 \mathrm{msec}$ ). The occurrence of the $\mathrm{AB}$ in this specific situation, however, was attributed to the destruction of the single letter stream by the long gap rather than to the temporal discrimination task.

Taken together, the results of Shapiro et al. (1994) and Sheppard et al. (2002) basically show that the processing of temporal stimulus properties as T1 task does not elicit an $\mathrm{AB}$. This result suggests that the processing of temporal stimulus properties differ from the processing of nontemporal properties. However, since these two studies have investigated T1 processing demands and have not examined temporal processing of stimuli presented during the $\mathrm{AB}$, they do not allow a conclusion about the influence of attention on temporal stimulus processing. To investigate the question whether decreased attentional availability during the $\mathrm{AB}$ results in a processing deficit for temporal processing or not, it is necessary to implement a temporal T2 task within the RSVP and measure the influence of T1 task processing on temporal performance for $\mathrm{T} 2$ task.

To our knowledge, there is only one study which has investigated temporal processing of T2 within the RSVP. This study (Visser \& Enns, 2001) employed a missing dot localization task (Hogben \& Di Lollo, 1974) as T2 task to examine whether temporal integration is modulated by the $\mathrm{AB}$. For this task, the authors constructed a $5 \times 5$ matrix and presented this matrix in two successive frames with a variable SOA. In addition to the central dot, one dot was randomly removed from one of the two matrix frames. The task of the participants was to indicate the location of the missing dot. Since there were several dots, the authors assumed that accurate localization can only be achieved by integrating the single frames into a single percept. The results indicated an effect of attention on temporal integration, namely that reduced attentional availability during the $\mathrm{AB}$ decreased the duration over which successive stimuli could be integrated. The authors interpreted this result as evidence that attended brief visual stimuli are visible for a longer period. In other words, attention prolongs visual persistence by reducing the temporal resolution of the visual system. This interpretation is in line with the above mentioned findings of Yeshurun and Levy (2003).

The study by Visser and Enns (2001), which investigates temporal processing within the $\mathrm{AB}$, supports the conclusion that attention prolongs visual persistence and thus reduces temporal resolution. There is, however, an alternative explanation of this result. This alternative takes into consideration that the dot localization task requires not only temporal integration but also spatial processing. 
Thus, it is possible that the results reflect the combined influence of attention on pattern perception and temporal integration. Therefore, by means of the dot localization task, it is not possible to clearly decide whether the processing cost during the $\mathrm{AB}$ results from a deficit in pattern perception or is in fact due to a lowered temporal integration. To circumvent possible interpretation problems and to investigate solely temporal stimulus processing, we used a temporal gap detection task (Yeshurun \& Levy, 2003). Since in this task no pattern perception is demanded, we aimed to investigate whether there is an $A B$ for temporal processing. We employed a modified RSVP paradigm and implemented a spatial task (Experiment 1) or a temporal gap detection task (Experiment 2) as T2 task within the RSVP. Whereas Experiment 1 served as control experiment, in Experiment 2 we investigated whether the $\mathrm{AB}$ influences temporal processing.

\section{EXPERIMENT 1}

In this first experiment we employed a spatial discrimination task to investigate whether T1 processing causes an AB. Since we aimed to avoid potential interference effects between the target tasks and the rapidly presented distractors in a typical RSVP, we used a skeletal RSVP (McLaughlin, Shore, \& Klein, 2001; Ward, Duncan, \& Shapiro, 1997). In this skeletal RSVP task, only T1 and $\mathrm{T} 2$ are presented and these two target stimuli are separated by a variable SOA. In addition, each target is followed by a mask. The results of this skeletal RSVP task reveal a similar pattern of results as a typical RSVP, and therefore one can assume that the same mechanisms contribute to the AB in both paradigms (McLaughlin et al., 2001; Ward et al., 1997). In addition, we aimed to assure that the proposed processing deficit of $\mathrm{T} 2$ is caused by the attentional processing of $\mathrm{T} 1$ and not by nonattentional factors, such as perceptual masking. To this end, we employed a singletask control condition in which the participants had to ignore $\mathrm{T} 1$ and only to respond to T2. Since no attentional processing of $\mathrm{T} 1$ is required in this control condition, no processing deficit should occur for $\mathrm{T} 2$.

\section{Method}

Participants. Eighteen adults served as paid participants. Two participants were excluded from further data analysis, because their recognition performance of $\mathrm{T} 1$ in the single-task was lower than $60 \%$. The remaining sixteen participants were 19-28 years old.

Stimuli and Apparatus. Stimulus presentation is illustrated in Figure 1. All stimuli were presented in white $\left(110 \mathrm{~cd} / \mathrm{m}^{2}\right)$ on a dark blue background $\left(2.5 \mathrm{~cd} / \mathrm{m}^{2}\right)$ of a computer screen and they were viewed from a distance of approximately $50 \mathrm{~cm}$. The stimuli consisted of a warning signal (asterisk with a diameter of $1.15^{\circ}$ angle of vision), the first target letters ( $\mathrm{G}, \mathrm{H}, \mathrm{B}$, or $\mathrm{Q}, 0.57^{\circ}$ angle of vision), a mask of scrambled letters $\left(2.29^{\circ} \times 2.29^{\circ}\right.$ angle of vision $)$, a random dot mask $\left(2.29^{\circ} \times 2.29^{\circ}\right.$ angle of vision $)$, and a Landolt square $\left(1.15^{\circ} \times 1.15^{\circ}\right.$ angle of vision $)$, which had a small gap $\left(0.12^{\circ}\right.$ angle of vision) on either the right or the left side. To reduce spatial uncertainty, all stimuli were presented within a small quadratic frame $\left(2.52^{\circ} \times 2.52^{\circ}\right.$ angle of vision $)$ at the center of the monitor. There were separate response panels for each hand.

Design and Procedure. A trial began with the presentation of the warning signal for $100 \mathrm{msec}$. After a variable foreperiod duration of 600,800 , or $1,000 \mathrm{msec}$, one of the four possible first target letters was presented for $16 \mathrm{msec}$. The target presentation was followed by a blank interval of $16 \mathrm{msec}$, and then the letter mask appeared for another $66 \mathrm{msec}$. The Landolt square was presented 83,266 , or 633 msec after letter mask offset (resulting in 183, 366, and $733 \mathrm{msec}$ SOA, respectively) and remained on the screen for $50 \mathrm{msec}$ before being masked. The mask terminated with the response or after $3 \mathrm{sec}$. Participants were asked to press the left key with their left index finger, if the gap of the Landolt square appeared on the left side or the right key with their right index finger, if the gap appeared on the right side. They were instructed to respond as accurate and as fast as possible. In the dual-task condition, a question mark signalled the participants to indicate which target letter

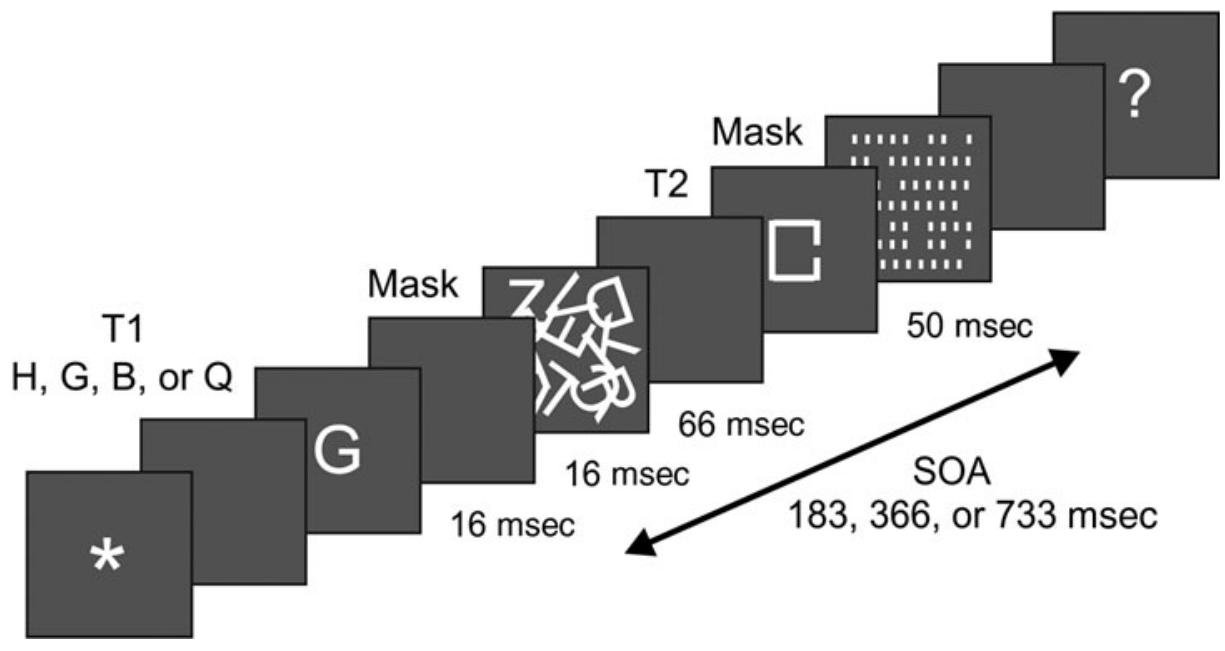

Figure 1. Stimulus sequence during the skeletal rapid serial visual presentation task in Experiment 1 . All stimuli were presented at the same spatial position. In the dual-task condition, participants had to identify the target letter (T1) and discriminate whether the gap of the Landolt square (T2) appeared on the left side or right side. A question mark prompted the participants to indicate which target letter they recognized. In the single-task condition, participants ignored T1 and responded only to $\mathrm{T} 2$. 
they had recognized. For this task, they responded by keypresses with their middle and index fingers. In the single-task condition, no question mark appeared. Participants initiated the next trial by pressing one of the response keys. A single session lasted about $1.5 \mathrm{~h}$ and consisted of 14 blocks of 24 trials each. The single-task and dual-task conditions were blocked and the order of conditions was counterbalanced across participants. The first two blocks of each condition were considered practice and discarded from data analysis. After each block, participants received feedback concerning the percentage of correct responses. The experiment factorially combined task (single-task vs. dual-task), SOA (183, 366, or $733 \mathrm{msec}$ ), and gap position (left vs. right).

Data analysis. Separate two-way ANOVAs with factors task and SOA were performed on percent correct discrimination performance of $\mathrm{T} 2$ and on mean RT of correct T2 responses (given correctly identified T1 in the dual-task condition). To assess possible interference effects of T2 processing on the processing of T1, we conducted an additional ANOVA with factor SOA on percent correct recognition performance of 11 in the dual task condition. Whenever appropriate, $p$ values were adjusted for violations of the sphericity assumption using the Huynh-Feldt correction. RTs shorter than 150 or greater than $1,500 \mathrm{msec}$ were considered outliers and their corresponding trials were discarded $(1.9 \%)$.

\section{Results and Discussion}

The overall recognition performance of T1 $(89.4 \%)$ was not influenced by SOA $(F<1)$, indicating that T2 presentation did not affect $\mathrm{T} 1$ processing. The results for the spatial discrimination task are summarized in Figure 2,

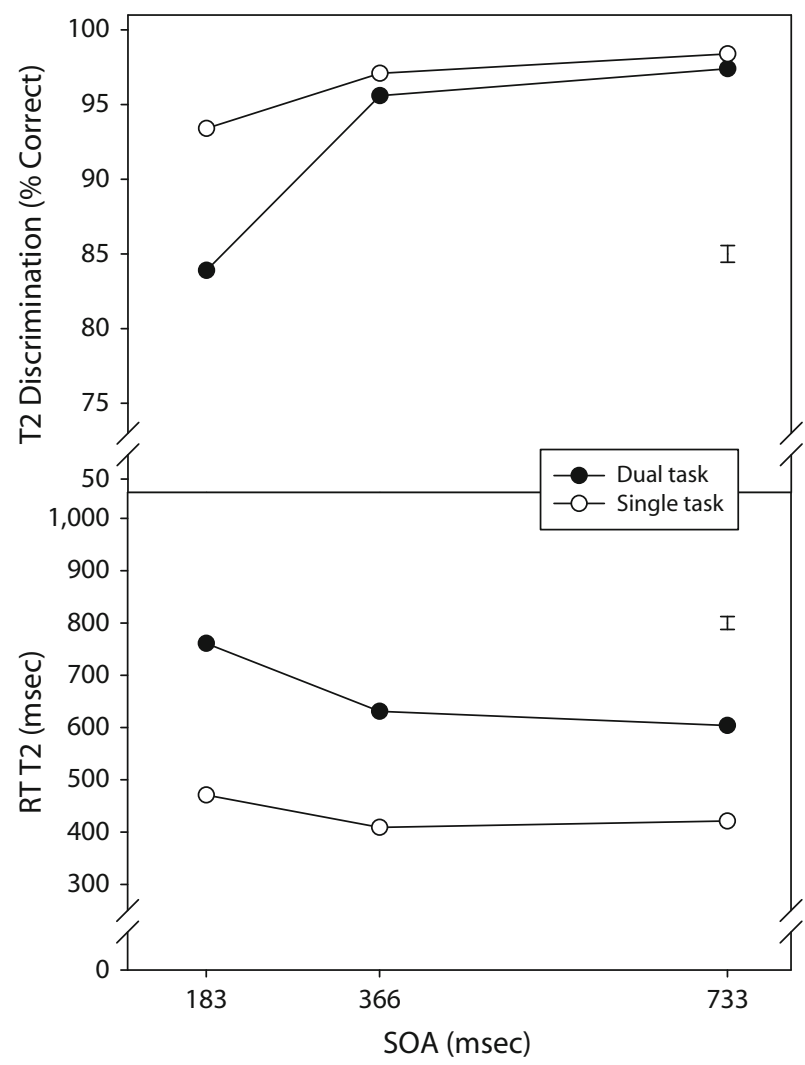

Figure 2. Results of Experiment 1 (spatial task). Percentage of correct $\mathrm{T} 2$ discrimination and mean $\mathrm{RT}$ as a function of task and SOA. The standard error was computed from the pooled error terms of the corresponding ANOVA according to a suggestion made by Loftus (2002). which depicts percent correct $\mathrm{T} 2$ discrimination and mean RT as a function of task and SOA. Discrimination performance increased with increasing SOA $[F(2,30)=25.8$, $p<.001]$. As expected, discrimination performance of the Landolt-squares' gap was better in the single-task than in the dual-task condition $[F(1,15)=31.3, p<.001]$. Most important, however, this task effect was clearly influenced by SOA $[F(2,30)=12.7, p<.001]$. As SOA increased, discrimination performance of the tasks converged to the same performance level. This interaction also mirrors the different effects of SOA in the two task conditions. There was a stronger influence of SOA in the dual-task $[F(2,30)=23.8, p<.001]$ than in the single$\operatorname{task}[F(2,30)=12.4, p<.001]$ condition. The interaction between task and SOA is consistent with previous studies (e.g., McLaughlin et al., 2001; Ward et al., 1997) and strongly suggests that an $\mathrm{AB}$ occurred for the spatial discrimination task within the skeletal presentation stream.

An ANOVA on RT supports this conclusion. Due to higher task demands, mean RT was slower in the dualtask than in the single-task condition $[F(1,15)=30.2$, $p<.001]$. Moreover, mean RT decreased with increasing SOA $[F(2,30)=54.5, p<.001]$, and this SOA effect was more pronounced in the dual-task than in the single-task condition $[F(2,30)=26.3, p<.001]$. This interaction most probably illustrates the time demands to process and consolidate T1 into a stable short-term memory representation. During this time interval, the processing of T2 is postponed and has to wait until attentional capacity is free for the second task. Since the participants were instructed to react as correctly and as fast as possible, one might argue they have strategically traded speed against accuracy. However, the present pattern of results - that is, slower RTs, together with an impaired discrimination performance for T2 at short SOA-excludes such a speedaccuracy trade-off account.

In summary, the present results confirm the wellknown $\mathrm{AB}$ effect for a spatial task. If participants have to process two rapidly presented targets which are subject to subsequent masking, the second target suffers from the attentional processing of $\mathrm{T} 1$. It is important to mention, however, that the processing deficit for $\mathrm{T} 2$ in the dual-task condition does not result from low-level masking. This conclusion is supported by the fact that $\mathrm{T} 2$ processing in the single-task condition stayed relatively constant across SOA. Taken together, the results of Experiment 1 are in line with several other studies showing an $\mathrm{AB}$ for different nontemporal stimuli (e.g., Chun \& Potter, 1995; Raymond et al., 1992).

\section{EXPERIMENT 2}

In this experiment, we embedded a temporal gap discrimination task (Yeshurun \& Levy, 2003) as T2 task within the RSVP to investigate the influence of attention on temporal discrimination performance. ${ }^{1}$

\section{Method}

Participants. A fresh sample of twenty-seven 19-43-year-old adults served as paid participants. As in Experiment 1, participants 
with a recognition performance of $\mathrm{T} 1$ in the single-task lower than $60 \%$ were excluded (seven participants).

Stimuli, Apparatus, Design, and Procedure. These were identical to the previous experiment except for the substitution of the spatial Landolt-square task by a temporal gap detection task (Figure 3$)$. Either a continuous white $\operatorname{dot}\left(0.2^{\circ}\right.$ angle of vision $)$ was presented for $116 \mathrm{msec}$ or the dot was interrupted by a blank interval of $16 \mathrm{msec}$. Participants were asked to judge, whether there was a continuous dot or a temporal gap in the presentation of the dot. They pressed the left key, if they perceived no gap and the right key, if they perceived a gap. The experiment comprised again 14 blocks of 24 trials each and the first two blocks of each condition (single-task, dual-task) were again considered practice and excluded from data analysis. Each block consisted of equal probable combinations of SOA $(183,366$, or $733 \mathrm{msec}$ ) and gap (gap vs. no gap).

Data analysis. Analogous to Experiment 1, an ANOVA with the factor SOA was conducted for T1 recognition performance. T2 discrimination performance and RT to $\mathrm{T} 2$ were investigated by ANOVAs with the factors task and SOA. As before, RTs shorter than 150 or greater than $1,500 \mathrm{msec}$ were considered outliers, and the corresponding trials were discarded (5.7\% of all trials).

\section{Results and Discussion}

As in Experiment 1, the overall recognition performance of T1 $(88.4 \%)$ was not influenced by SOA $(F<1)$. Figure 4 summarizes the results of the temporal discrimination task. In line with the results of Experiment 1, discrimination performance of T2 was better in the singletask than in the dual-task condition $[F(1,19)=11.4, p<$ $.01]$. Overall discrimination performance of the temporal gap detection did not change depending on SOA $(p>$ .18). Theoretically important, however, task and SOA interacted $[F(2,38)=5.1, p<.05]$. As SOA increased, the difference in discrimination performance between singletask and dual-task vanished. This result strongly suggests that an $\mathrm{AB}$ occurred for the temporal discrimination task. To strengthen this conclusion, additional analyses were performed separately on recognition performance in the single-task and the dual-task. These analyses revealed an influence of SOA in the dual-task condition $[F(2,38)=$ $4.1, p<.05]$ that was absent in the single-task condition $(F<1)$

An ANOVA of RTs revealed a similar pattern of results as in Experiment 1. Mean RT was slower in the dual-task than in the single-task condition $[F(1,19)=43.9, p<$ $.001]$. Furthermore, mean RT decreased with increasing SOA $[F(2,38)=38.0, p<.001]$, but the interaction between factors task and SOA shows that this SOA effect was more pronounced in the dual-task than in the singletask condition $[F(2,38)=23.7, p<.001]$.

Taken together, both $\mathrm{T} 2$ discrimination performance and RT revealed a similar pattern of results as in Experiment 1 . Theoretically most important, however, even for the temporal gap detection task, an AB occurred. As in Experiment 1, it can be excluded that the $\mathrm{T} 2$ processing deficit is caused by low-level masking factors since performance in the single-task condition remained at a high constant performance level. If the T2 processing deficit would have been caused by masking, the deficit should have occurred in the single-task as well as in the dual-task condition. Therefore, analogous to spatial discrimination, temporal discrimination performance suffers if attention is not allocated to this task due to $\mathrm{T} 1$ processing

\section{GENERAL DISCUSSION}

This study examined the influence of attention on temporal stimulus processing. Specifically, we assessed whether there is any influence of attentional allocation on temporal discrimination performance at a relatively late processing level. A skeletal RSVP task was used to address this question. In Experiment 1, we employed a spatial task, whereas in Experiment 2, we required the participants to perform a temporal gap detection task. The results were clear. First, a typical AB was observed for

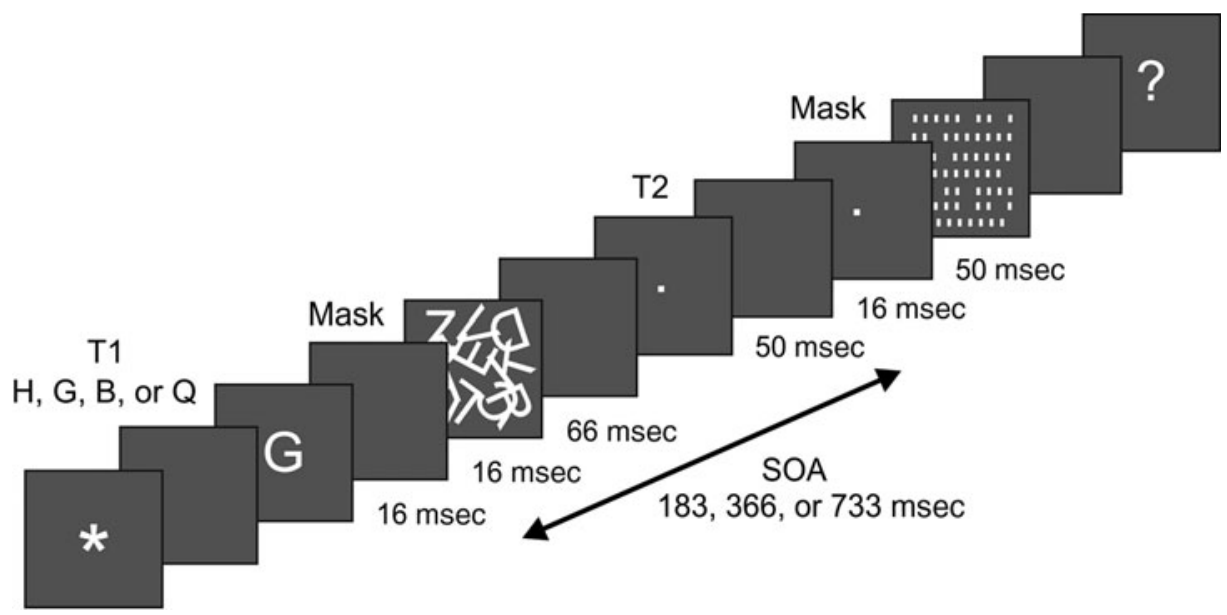

Figure 3. Stimulus sequence during the skeletal rapid serial visual presentation task in Experiment 2 . In the dual-task condition, participants had to identify the target letter (T1) and discriminate whether there was a temporal gap in the presentation of the dot (T2). A question mark signaled the participants to indicate which target letter they recognized. In the single-task condition, participants ignored $\mathrm{T} 1$ and responded only to $\mathrm{T} 2$. The figure shows the situation in which a temporal gap of 16 msec occurred in the dot presentation. 


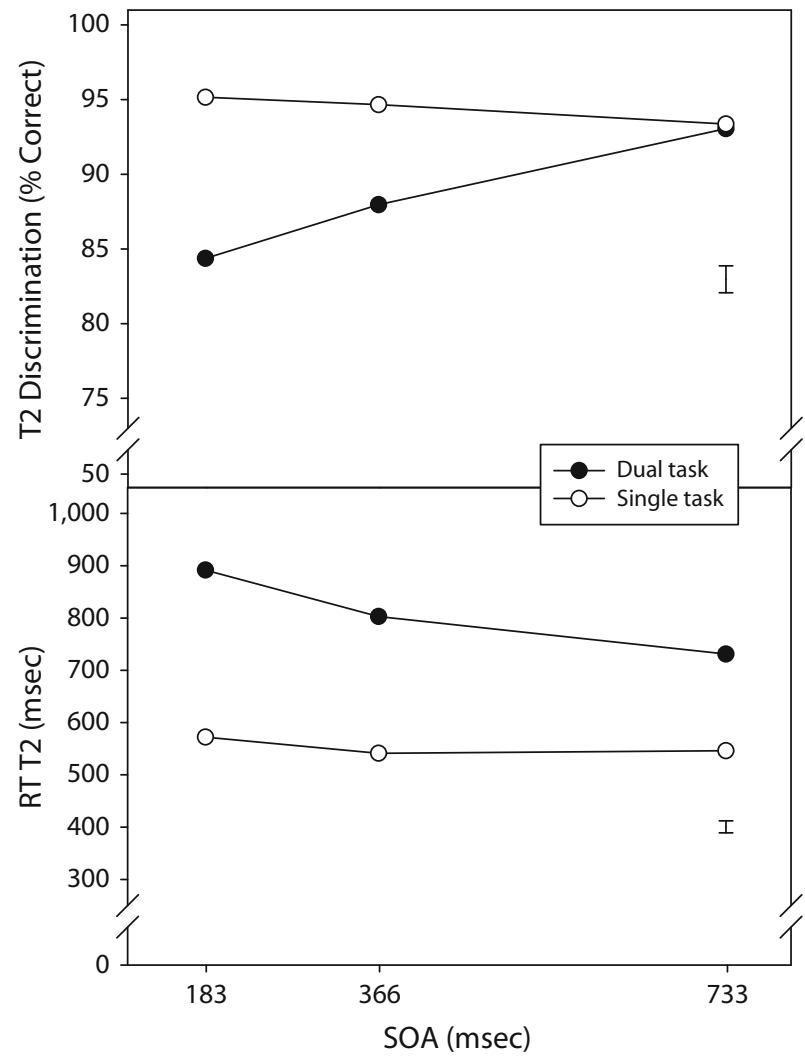

Figure 4. Results of Experiment 2 (temporal task). Percentage of correct $\mathrm{T} 2$ discrimination and mean $\mathrm{RT}$ as a function of task and SOA. The standard error was computed from the pooled error terms of the corresponding ANOVA according to a suggestion made by Loftus (2002).

the spatial task. This result agrees with several other studies reporting an $\mathrm{AB}$ for different nontemporal stimulus properties (Broadbent \& Broadbent, 1987; Chun \& Potter, 1995; Luck et al., 1996; Raymond et al., 1992; Rolke et al., 2001; Ross \& Jolicœur, 1999; Shapiro et al., 1997). In addition, this result clearly confirms the usability of the skeletal RSVP paradigm to modulate attentional processing demands (see also McLaughlin et al., 2001; Ward et al., 1997). Second, and of theoretical importance, the results of Experiment 2 revealed an $\mathrm{AB}$ also for temporal gap discrimination performance - that is, temporal discrimination was impaired when attentional resources were decreased. Therefore, the reduced allocation of attention in this paradigm hampers the processing of temporal stimulus properties.

The overall pattern of results - an AB for temporal discrimination in this study and the decreased temporal integration during the $\mathrm{AB}$ in the study of Visser and Enns (2001) — seems to support the assumption that reduced attentional availability during the $\mathrm{AB}$ impairs temporal stimulus processing. The interpretation of the two results, however, assumes that there are two different mechanisms. Whereas Visser and Enns interpreted their results as evidence that attention prolongs the persistence of brief visual stimuli, the present results support the opposite conclusion. Specifically, the present results show a decreased probability of detecting a short temporal gap within a single stimulus when attentional resources are reduced during the $\mathrm{AB}$ and a recovery of discrimination performance with increasing attentional availability outside the $\mathrm{AB}$ time epoch. Since the detection of the temporal gap relies on the temporal resolution of the visual system, this pattern of results indicates that the temporal resolution of the visual system decreases when the stimulus receives no attentional resources. In other words, according to the present results attention seems to increase rather than decrease the temporal resolution of the visual system.

One possible way to account for this discrepancy is to assume that the different tasks, such as the dot localization and temporal gap detection tasks, require different processing mechanisms that might contribute to the results. As already mentioned in the introduction, the dot localization task can not be performed without spatial processing because the locus of the missing dot must be reported. The authors have noticed that the effects of their experiments might be due to pattern perception and thus, they tried to weaken pattern processing and instead of the dot localization task they required a missing dot detection task in another experiment (Visser \& Enns, 2001). They found a lower accuracy of missing dot detection within the AB. Although the authors argue that this result points more clearly to an attentional effect which is specific to temporal integration, this dot detection task does not totally exclude spatial and pattern processing of the targets. Therefore, the results of Visser and Enns (2001) leave open the possibility that the attentional effects were, at least in part, due to pattern processing of the dot matrices. This assumed impairment of pattern processing, however, would be in line with $\mathrm{AB}$ research showing decreased performance for nontemporal stimulus properties within the AB (Arnell \& Jolicœur, 1999; Arnell \& Larson, 2002; Chun \& Potter, 1995; Dell'Acqua et al., 2001; Luck, et al., 1996; Raymond et al., 1992; Rolke et al., 2001; Ross \& Jolicœur, 1999; Shapiro et al., 1997). In the present study, we excluded pattern processing of T2 and employed a temporal gap detection task. Since this task solely relies on temporal processing, it can not benefit from any influence of attention on pattern processing. We found an $A B$ for the temporal gap detection performance and thus, our results suggest that decreased attentional availability impairs the processing of temporal stimulus attributes.

The impairment of the temporal gap detection task by lowered attention resources during the $\mathrm{AB}$ in the present study comes along with the opposite effect in the study of Yeshurun and Levy (2003) and Rolke et al. (in press). In these studies, an enhanced temporal discrimination performance resulted when transient attention was spatially allocated away from stimulus location. One way to account for these differential effects of attention on temporal processing might be that a different mode of attention operates within each of the two paradigms. Specifically, these different paradigms presumably are associated with attentional modulations at different visual processing levels. In fact, Yeshurun and Levy oriented attention by an exogenous cue toward a specific spatial location within the visual field. Within an exogenous cuing paradigm, at- 
tention most probably exerts its influence on visual stimulus processing at early processing levels within the visual system. This assumption is supported by several electrophysiological studies that show an attentional modulation of early evoked potentials (e.g., Luck \& Girelli, 1998; Luck et al., 1994; Mangun \& Hillyard, 1991).

In the present study, we temporally oriented attention away from stimulus processing at a foveal location. The $A B$, however, seems to be a processing deficit that emerges at a postperceptual processing level. As outlined in the introduction, this late selection view of the $A B$ is proved by several lines of evidence. For example, early evoked potentials are not suppressed during the $\mathrm{AB}$ (Vogel et al., 1998). In addition, the late locus of the $A B$ processing deficit is further supported by the presence of a crossmodal AB (Arnell \& Jolicœur, 1999; Arnell \& Larson, 2002; Dell'Acqua et al., 2001) and the demonstration of a semantic processing within the AB (Luck et al., 1996; Maki et al., 1997; Rolke et al., 2001; Shapiro et al., 1997; Vogel et al., 1998). The pattern of results in the present study shows an $\mathrm{AB}$ for the temporal discrimination task; discrimination performance was impaired if attentional resources were reduced for target processing. Therefore, one way to interpret the divergent results obtained within the present $\mathrm{AB}$ paradigm and within the spatial cuing paradigm of Yeshurun and Levy (2003) and Rolke et al. (in press) is to assume that attention exerts a differential effect on the temporal resolution of the visual system at different processing levels.

This idea that attention might tap different levels of processing and thus exerts differential effects on temporal discrimination performance is compatible with the mechanism Yeshurun and Levy (2003) have suggested for their surprising results, that attention enhances the spatial yet lowers the temporal resolution of the visual system. The authors (2003; see also Yeshurun, 2004) have proposed a neurophysiological hypothesis of attention and hypothesized that attention selectively influences the parvocellular and the magnocellular paths of the visual system. More specifically, Yeshurun and Levy have assumed that visual attention facilitates the activity of parvocellular neurons at the attended location, which in turn inhibits the activity of magnocellular neurons at the same location. This proposed attentional mechanism highlights the attributes of the parvocellular system compared to those of the magnocellular system. Neurophysiological studies have shown that parvocellular neurons not only exhibit smaller receptive fields but also longer response durations than magnocellular neurons (e.g., Maunsell \& Gibson, 1992; Schmolesky et al., 1998). Thus, Yeshurun and Levy have argued that the increased activity of parvocellular neurons at the attended location enhances the spatial resolution. Crucially, however, the associated prolonged neuronal response minimizes the possibility to detect the temporal gap of the flickering target stimulus at the attended location, since stimulus input at the attended location is integrated over a longer period.

Converging evidence for the neurophysiological mechanism and the assumption that spatial attention might delay the perceived offset of a stimulus is provided by a recent
RT study of Rolke, Ulrich, and Bausenhart (2006). We tested this assumption in a spatial cuing paradigm similar to the one employed by Yeshurun and Levy (2003), but asked participants to provide a speeded response to stimulus offset. According to the hypothesis of Yeshurun and Levy, we expected longer RTs in the attended rather than in the neutral condition. Consistent with this expectation, offset RTs were prolonged for attended compared to unattended stimuli. Thus, the results directly bear up the proposal made by Yeshurun and Levy.

The neurophysiological hypothesis is in line with several results emerging from spatial attention tasks. Moreover, the hypothesis provides an interesting account to explain the negative effect of attention on temporal processing. Most important for the present study, however, it is very likely that this proposed mechanism of attention on parvocellular and magnocellular neurons is restricted to early processing levels. One hint for this assumption comes from a recent study of Hein et al. (2006). These authors used different spatial cues to orient spatial attention to a stimulus location and employed a temporal-order discrimination task to investigate the influence of attention on temporal stimulus processing. Whereas exogenous cues impaired discrimination performance, endogenous cues facilitated temporal-order discrimination. The results therefore indicate that automatic but not voluntary shifts of attention lower the temporal sensitivity of the visual system. In line with other work (Briand, 1998; Briand \& Klein, 1987; Klein, 1994; Klein, Kingstone, \& Pontefract, 1992; Riggio \& Kirsner, 1997), Hein et al. concluded that automatic and voluntary shifts of attention act at different levels within the visual system. Specifically, automatically but not voluntarily controlled attention seems to be involved in early or middle visual processing stages (Briand \& Klein, 1987; Nakayama \& Mackeben, 1989; Riggio \& Kirsner, 1997). Therefore, the study of Hein et al. (2006) supports the assumption that attention impairs the temporal resolution of the visual system at an early level, but facilitates temporal resolution at a later level.

In contrast to the differential effect of transient spatial attention on temporal and spatial stimulus properties reported by Yeshurun and Levy (2003), the results of the present study revealed a similar performance decrement for both the temporal task and the spatial task if attentional resources were unavailable during the $\mathrm{AB}$. Thus, this pattern of results implies that attention exerts a comparable influence on both stimulus attributes at a later processing level. For example, one might assume that attention influences spatial as well as temporal representations by signal enhancement (see, e.g., Pashler, 1998). In the context of the $\mathrm{AB}$, signal enhancement of $\mathrm{T} 2$ representation might be equivalent to an increased probability of $\mathrm{T} 2$ consolidation into a stable short-term memory representation. This possible mode of attention might affect stimuli at a rather late processing level, and thus the receptor based mechanism proposed by the neurophysiological hypothesis might remain ineffective.

Although the possibility that the differential effects of attention on temporal stimulus processing within the exogenous spatial attention studies of Yeshurun and Levy 
(2003) and Hein et al. (2006) and the present AB study might be explained by the allocation of attention to different processing levels, we can not be completely sure whether this is the only explanation. It is also quite possible, however, that entirely different mechanisms operate at early and late processing levels and this might lead to the different influences of attention on temporal stimulus features at early and late processing levels. For example, whereas spatially allocated attention at an early processing level might evoke facilitatory as well as inhibitory mechanisms (Bennett \& Pratt, 2001; Dosher \& Lu, 2000), the decrement of attentional processing resources at a late processing level during the $\mathrm{AB}$ might result only in an attenuation of stimulus processing at the same spatial location. Thus, further research is necessary to address the question which specific mechanisms contribute to the influence of attention on temporal stimulus processing within the visual system and whether these mechanisms exert their influences on different processing levels.

In conclusion, the present study examined whether the negative effect of attention on temporal discrimination reported by Yeshurun and Levy (2003) might be a common phenomenon of visual attention or whether this effect might be restricted to spatial attention. We employed an RSVP paradigm in which attention is temporally not available for target processing. The results revealed a comparable $\mathrm{AB}$ for the processing of spatial and temporal stimulus properties. Thus, if attentional demands are modulated at a late processing level, temporal stimulus processing is impaired by reduced attentional resources. We hypothesize that attention exerts a differential influence on temporal stimulus processing at different processing levels.

\section{AUTHOR NOTE}

This study was supported by Grant RO 3034/1-1 of the German Research Foundation (DFG). We thank Elisabeth Hein and Allen Osman for helpful comments. Correspondence concerning this article should be sent to B. Rolke, Cognitive and Biological Psychology, University of Tübingen, Friedrichstrasse 21, 72072 Tübingen, Germany (e-mail: bettina.rolke@ uni-tuebingen.de).

\section{REFERENCES}

Arnell, K. M., \& Joliceeur, P. (1999). The attentional blink across stimulus modalities: Evidence for central processing limitations. Journal of Experimental Psychology: Human Perception \& Performance, 25, 630-648.

ARnell, K. M., \& LARson, J. M. (2002). Cross-modal attentional blinks without preparatory task-set switching. Psychonomic Bulletin \& Review, 9, 497-506.

Bennett, P. J., \& Pratt, J. (2001). The spatial distribution of inhibition of return. Psychological Science, 12, 76-80.

Bonnel, A.-M., PossamaÏ, C.-A., \& Schmitt, M. (1987). Early modulation of visual input: A study of attentional strategies. Quarterly Journal of Experimental Psychology, 39A, 757-776.

BRIAND, K. A. (1998). Feature integration and spatial attention: More evidence of a dissociation between endogenous and exogenous orienting. Journal of Experimental Psychology: Human Perception \& Performance, 24, 1243-1256.

BRiand, K. A., \& Klein, R. M. (1987). Is Posner's "beam" the same as Treisman's "glue"? On the relation between visual orienting and feature integration theory. Journal of Experimental Psychology: Human Perception \& Performance, 13, 228-241.

Broadbent, D. E. (1958). Perception and communication. London: Pergamon,
Broadbent, D. E., \& Broadbent, M. H. (1987). From detection to identification: Response to multiple targets in rapid serial visual presentation. Perception \& Psychophysics, 42, 105-113.

Cheal, M. L., Lyon, D. R., \& Hubbard, D. C. (1991). Does attention have different effects on line orientation and line arrangement discrimination? Quarterly Journal of Experimental Psychology, 43A, 825-857.

Chun, M. M., \& Potter, M. C. (1995). A two-stage model for multiple target detection in rapid serial visual presentation. Journal of Experimental Psychology: Human Perception \& Performance, 12, 109-127.

Crebolder, J. M., Jolicceur, P., \& McIlwaine, J. D. (2002). Loci of signal probability effects and the attentional blink bottleneck. Journal of Experimental Psychology: Human Perception \& Performance, 28, 695-716.

Dell'Acqua, R., Turatto, M., \& Jolicceur, P. (2001). Cross-modal attentional deficits in processing tactile stimulation. Perception \& Psychophysics, 63, 777-789.

Dosher, B. A., \& LU, Z.-L. (2000). Noise exclusion in spatial attention. Psychological Science, 11, 139-146.

DownING, C. J. (1988). Expectancy and visual-spatial attention: Effects on perceptual quality. Journal of Experimental Psychology: Human Perception \& Performance, 14, 188-202.

Hein, E., Rolke, B., \& UlRich, R. (2006). Visual attention and temporal discrimination: Differential effects of automatic and voluntary cueing. Visual Cognition, 13, 29-50.

Henderson, J. M. (1991). Stimulus discrimination following covert attentional orienting to an exogenous cue. Journal of Experimental Psychology: Human Perception \& Performance, 17, 91-106.

Henderson, J. M. (1996). Spatial precues affect discrimination in the absence of visual noise. Journal of Experimental Psychology: Human Perception \& Performance, 22, 780-787.

Hogben, J. H., \& Di Lollo, V. (1974). Perceptual integration and perceptual segregation of brief visual stimuli. Vision Research, 14, 10591069.

IsAak, M. I., Shapiro, K. L., \& Martin, J. (1999). The attentional blink reflects retrieval competition among multiple rapid serial visual presentation items: Tests of an interference model. Journal of Experimental Psychology: Human Perception \& Performance, 25, 1774-1792.

Jolicceur, P., Dell'Acqua, R., \& Crebolder, J. M. (2001). The attentional blink bottleneck. In K. [L.] Shapiro (Ed.), The limits of attention: Temporal constraints in human information processing (pp. 82-99). Oxford: Oxford University Press.

KLeIN, R. M. (1994). Perceptual-motor expectancies interact with covert visual orienting under conditions of endogenous but not exogenous control. Canadian Journal of Experimental Psychology, 48, 167-181.

Klein, R. [M.], Kingstone, A., \& Pontefract, A. (1992). Orienting of visual attention. In K. Rayner (Ed.), Eye movements and visual cognition: Scene perception and reading (pp. 46-65). New York: Springer.

Loftus, G. R. (2002). Analysis, interpretation, and visual presentation of experimental data. In H. [E.] Pashler (Ed.), Stevens' Handbook of experimental psychology (3rd ed.): Vol. 4. Methodology in experimental psychology (pp. 339-390). New York: Wiley.

LUCK, S. J., \& GIRELLI, M. (1998). Electrophysiological approaches in the study of selective attention in the human brain. In R. Parasuraman (Ed.), The attentive brain (pp. 71-94). Cambridge, MA: MIT Press.

Luck, S. J., Hillyard, S. A., Mouloua, M., Woldorff, M. G., Clark, V. P., \& HAwKINS, H. L. (1994). Effects of spatial cuing on luminance detectability: Psychophysical and electrophysiological evidence for early selection. Journal of Experimental Psychology: Human Perception \& Performance, 20, 887-904.

Luck, S. J., Vogel, E. K., \& Shapiro, K. L. (1996). Word meanings can be accessed but not reported during the attentional blink. Nature, 383, 616-618.

Maki, W. S., Frigen, K., \& Paulson, K. (1997). Associative priming by targets and distractors during rapid serial visual presentation: Does word meaning survive the attentional blink? Journal of Experimental Psychology: Human Perception \& Performance, 23, 1014-1034.

Mangun, G. R., \& Hillyard, S. (1991). Modulations of sensoryevoked brain potentials indicate changes in perceptual processing during visual-spatial priming. Journal of Experimental Psychology: Human Perception \& Performance, 17, 1057-1074.

Maunsell, J. H., \& Gibson, J. R. (1992). Visual response latencies in 
striate cortex of the macaque monkey. Journal of Neurophysiology, 68, 1332-1344.

McLaughlin, E. N., Shore, D. I., \& Klein, R. M. (2001). The attentional blink is immune to masking-induced data limits. Quarterly Journal of Experimental Psychology, 54A, 169-196.

Nakayama, K., \& Mackeben, M. (1989). Sustained and transient components of focal visual attention. Vision Research, 29, 1631-1647.

NeIsser, U. (1967). Cognitive psychology. New York: AppletonCentury-Crofts.

Pashler, H. E. (1998). The psychology of attention. Cambridge, MA: MIT Press.

Pashler, H. [E.], \& Johnston, J. C. (1989). Chronometric evidence for central postponement in temporal overlapping tasks. Quarterly Journal of Experimental Psychology, 41A, 19-45.

Posner, M. I., SNyder, C. R., \& Davidson, B. J. (1980). Attention and the detection of signals. Journal of Experimental Psychology: General, 109, 160-174.

Raymond, J. E., Shapiro, K. L., \& Arnell, K. M. (1992). Temporary suppression of visual processing in an RSVP task: An attentional blink? Journal of Experimental Psychology: Human Perception \& Performance, 18, 849-860.

Raymond, J. E., Shapiro, K. L., \& Arnell, K. M. (1995). Similarity determines the attentional blink. Journal of Experimental Psychology: Human Perception \& Performance, 21, 653-662.

Riggio, L., \& Kirsner, K. (1997). The relationship between central cues and peripheral cues in covert visual orientation. Perception \& Psychophysics, 59, 885-899.

Rolke, B., Dinkelbach, A., Hein, E., \& Ulrich, R. (in press). Does attention impair temporal discrimination? Examining nonattentional accounts. Psychological Research.

Rolke, B., Heil, M., Streb, J., \& Hennighausen, E. (2001). Missed prime words within the attentional blink evoke an N400 semantic priming effect. Psychophysiology, 38, 165-174.

Rolke, B., Ulrich, R., \& Bausenhart, K. M. (2006). Attention delays perceived stimulus offset. Vision Research, 46, 2926-2933

Ross, N. E., \& Jolicceur, P. (1999). Attentional blink for color. Journal of Experimental Psychology: Human Perception \& Performance, 25, 1483-1494.

Ruthruff, E., \& Pashler, H. [E.] (2001). Perceptual and central interference in dual-task performance. In K. [L.] Shapiro (Ed.), The limits of attention: Temporal constraints in human information processing (pp. 100-123). Oxford: Oxford University Press.

Schmolesky, M. T., Wang, Y., Hanes, D. P., Thompson, K. G., Leutgeb, S., Schall, J. D., \& Leventhal, A. G. (1998). Signal timing across the macaque visual system. Journal of Neurophysiology, 79, 3272-3278.

Shapiro, K. L., Driver, J., Ward, R., \& Sorensen, R. E. (1997). Priming from the attentional blink: A failure to extract visual tokens but not visual types. Psychological Science, 8, 95-100.
Shapiro, K. L., Raymond, J. E., \& Arnell, K. M. (1994). Attention to visual pattern information produces the attentional blink in rapid serial visual presentation. Journal of Experimental Psychology: Human Perception \& Performance, 20, 357-371.

Sheppard, D. M., Duncan, J., Shapiro, K. L., \& Hillstrom, A. P. (2002). Objects and events in the attentional blink. Psychological Science, 13, 410-415.

Treisman, A. M. (1960). Contextual cues in selective listening. Quarterly Journal of Experimental Psychology, 12, 242-248.

VISSER, T. A., \& ENNS, J. T. (2001). The role of attention in temporal integration. Perception, 30, 135-145.

Vogel, E. K., \& LucK, S. J. (2002). Delayed working memory consolidation during the attentional blink. Psychonomic Bulletin \& Review, 9, 739-743.

Vogel, E. K., Luck, S. J., \& Shapiro, K. L. (1998). Electrophysiological evidence for a postperceptual locus of supression during the attentional blink. Journal of Experimental Psychology: Human Perception \& Performance, 24, 1656-1674.

WARD, R., DunCAN, J., \& Shapiro, K. [L.] (1997). Effects of similarity, difficulty, and nontarget presentation on the time course of visual attention. Perception \& Psychophysics, 59, 593-600.

YESHURUN, Y. (2004). Isoluminant stimuli and red background attenuate the effects of transient spatial attention on temporal resolution. Vision Research, 44, 1375-1387.

Yeshurun, Y., \& CARRAsco, M. (1998). Attention improves or impairs visual performance by enhancing spatial resolution. Nature, 396, $72-75$.

Yeshurun, Y., \& Carrasco, M. (1999). Spatial attention improves performance in spatial resolution tasks. Vision Research, 39, 293-306.

Yeshurun, Y., \& CARrasco, M. (2000). The locus of attentional effects in texture segmentation. Nature Neuroscience, 3, 622-627.

Yeshurun, Y., \& Levy, L. (2003). Transient spatial attention degrades temporal resolution. Psychological Science, 14, 225-231.

\section{NOTE}

1. One might assume that the negative influence of attention on temporal stimulus processing presupposes the allocation of attention at different locations in space. This assumption might be supported by the fact that, so far, the detrimental effect on temporal processing was found with manipulations of spatial attention. The results of Yeshurun and Levy (2003), however, argue against this assumption. The authors varied target eccentricity and provided evidence that the effect of attention is not restricted to peripheral locations, but is also present at the fovea.

(Manuscript received June 29, 2006; revision accepted for publication May 3, 2007. ) 